\title{
Characterization of Mycobacterium tuberculosis of Lebanese patients by double-repetitive-element polymerase chain reaction
}

\author{
M. Hamze, ${ }^{1}$ A. Rahmo ${ }^{2}$ and M. Saade 3
}

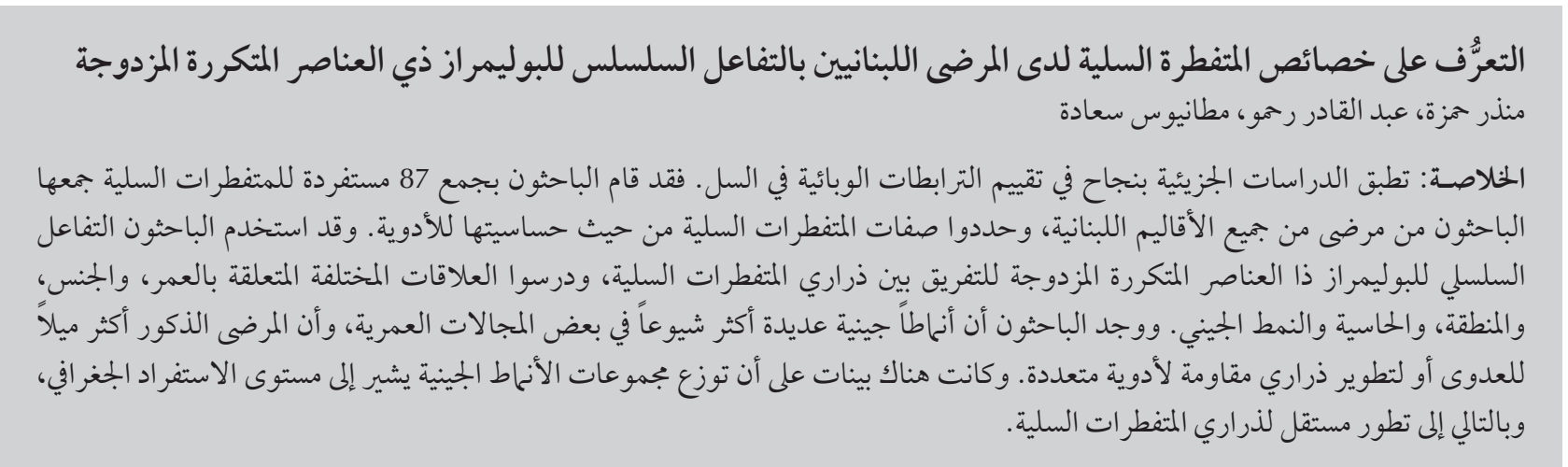

ABSTRACT Molecular studies have been successfully applied in evaluating epidemiological linkages in tuberculosis. A total of 87 isolates of Mycobacterium tuberculosis were collected from patients in all regions of Lebanon and characterized in terms of drug sensitivity. Double-repetitive-element polymerase chain reaction was used to differentiate between strains. Various correlations related to age, sex, region, sensitivity and genotype were examined. Several genotypes were more common in certain age ranges. Male patients appeared more likely either to be infected by or to develop multi-drug resistant strains. There was also evidence for a distribution of genotype groups indicating some level of geographical isolation and hence separate evolution of M. tuberculosis strains.

\section{Caractérisation de Mycobacterium tuberculosis chez des patients libanais par PCR d'éléments répétitifs doubles}

RÉSUMÉ Des études moléculaires ont été utilisées avec succès pour évaluer les liens épidémiologiques de la tuberculose. Au total, 87 isolats de Mycobacterium tuberculosis issus de patients de toutes les régions du Liban ont été recueillis et caractérisés en termes de sensibilité aux médicaments. Une PCR d'éléments répétitifs doubles (DRE-PCR) a été utilisée afin de différencier les souches. Différentes corrélations relatives à l'âge, au sexe, à la région, à la sensibilité et au génotype ont été étudiées. Certains génotypes étaient plus fréquents dans certaines tranches d'âge. II est apparu que les patients de sexe masculin présentaient un risque plus important de développer des souches polypharmacorésistantes ou d'être infectés par de telles souches. Des preuves de distribution des groupes de génotypes, indiquant un certain degré d'isolement géographique et donc une évolution différente des souches de M. tuberculosis, ont également été observées. 


\section{Introduction}

The spread of extensively drug-resistant tuberculosis (TB) poses a serious threat that could reverse recent achievements in TB control [1]. In particular, treatment of patients with multidrug resistant (MDR) strains is long and costly, and requires the use of drugs which frequently cause severe adverse reactions. The treatment outcome is poor, with low cure rates and high fatality ratios [2]. MDR TB cases may remain infectious in the community for a prolonged period of time. The emergence of socalled TB "hot-spots" around the world highlights the need for more research and application of current knowledge.

Molecular epidemiological studies have been successfully applied in evaluating epidemiological linkages in TB, and the discovery of unexpected ones. They were essential in distinguishing relapse from reinfection in recurrences of $\mathrm{TB}$ [3] in establishing evidence of laboratory cross-contaminations (false positives) [4] and in documenting the association of particular genotypes with hypervirulence and multi-resistance [5]. The importance of large-scale genotyping of Mycobacterium tuberculosis has prompted countries such as the United States of America to implement large-scale rapid genotyping of all local cases [6].

Various methods have been used for the genotyping of $M$. tuberculosis TB. The double-repetitive-element polymerase chain reaction (DRE-PCR) method is relatively fast, simple and cost-effective $[7,8]$. The technique serves as a tailored approach for preliminary genotyping and has the potential for efficient implementation in low-resource countries, where $\mathrm{TB}$ is generally widespread and endemic.

In this study we isolated various strains of MTB from Lebanese patients in various regions of the country, followed by characterization of their sensitivity to antibiotics and genotyping via a slightly modified DRE-PCR method.

\section{Methods}

\section{Sample}

Following an agreement with the National Tuberculosis Programme of the Lebanese Ministry of Health, sputum samples from newly-detected cases of pulmonary TB (84 samples) and from patients with a past history of the disease who were still under treatment (3 samples) were collected between April 2004 and October 2005 from all Lebanese provinces (muhafazat). Samples were sent refrigerated to the laboratory of the hospital at the Middle East Health Centre in Bsalim/Metin in Lebanon, accompanied by all related information. No information on the HIV status of the patients was obtained.

\section{Specimen preparation and culture}

Specimens were decontaminated by the $2 \% \mathrm{~N}$-acetyl-L-cysteine $\mathrm{NaOH}$ method. After neutralization and centrifugation, $0.2 \mathrm{~mL}$ of the concentrated specimen was inoculated onto a slant of Lowenstein-Jensen medium (Becton Dickinson Microbiology Systems, Cockeysville, Maryland); $0.5 \mathrm{~mL}$ was also inoculated into modified Middlebrook 7H9 broth (BD BBL MGIT, Becton-Dickinson) supplemented with PANTA antibiotic mixture (BBL MGIT PANTA, Becton-Dickinson) and OADC enrichment. The 2 media were incubated at $35-37^{\circ} \mathrm{C}$ and the Lowenstein-Jensen slant was examined for growth twice weekly. The BD BBL MGIT tube was read daily with UV light using a positive and a negative control. A positive tube was subcultured and an acid-fast smear prepared.

\section{Susceptibility testing}

Antibiotic susceptibility testing was performed using the BBL mycobacteria growth indicator tube (MGIT) AST system (Becton-Dickinson) based on comparing growth of the Mycobacterium strains in a drug-containing tube with that of a drug-free tube. The day after a positive MGIT tube became positive, final concentrations of antibiotics were added to MGIT tube: streptomycin (STR) $(0.8 \mu \mathrm{g} / \mathrm{mL})$,. isoniazid (INH) $(0.1 \mu \mathrm{g} / \mathrm{mL})$, rifampicin (RIF) $(1 \mu \mathrm{g} /$ $\mathrm{mL}$ and ethambutol (EMB) $(3.5 \mu \mathrm{g} /$ $\mathrm{mL}$ ). A control tube was set up for each antibiogram. A control strain of M. tuberculosis (H37 Rv, ACTCC 27294) susceptible to all standard antiTB drugs was included. The tubes were read with a $365 \mathrm{~nm}$ UV transilluminator on the third incubation day [9]. The patterns of antibiotic sensitivity $(S)$ and resistance (R) are described in the text using the notation RRSR, RRRS etc., with the following sequence: rifampin (RIF)-isoniazid (INH)-ethambutol (EMB)-streptomycin (STR).

\section{Identification}

Identity of strains was based on the following biochemical characteristics: production of niacin, nitrate reductase and catalase at laboratory temperature; production of catalase at $68^{\circ} \mathrm{C}$; hydrolysis of Tween 80 in 10 days, urease in 18 hours and arylsulfatase in 3 days [10].

\section{DRE-PCR procedure}

For DNA extraction, a loopful of each culture was suspended in ATL buffer $(0.2 \mathrm{~mL})$ and DNA was extracted using QIAamp DNA blood mini-kit (Qiagen). The manufacturer's procedure was adhered to except for the incubation period, which was extended to 3 hours. DNA was eluted in $100 \mu \mathrm{L}$ PCR water (Gibco). The DRE-PCR procedure was followed as reported by Harris [11], a modification of Friedman et al. [12]. The PCR amplification mixture contained $67 \mathrm{mM}$ tris (pH 8.8) $16 \mathrm{mM}$ $\left(\mathrm{NH}_{4}\right)_{2} \mathrm{SO}_{4^{\prime}} 0.01 \%$ tween $20(1 \times$ reaction buffer; Euroclone), $2.5 \mathrm{mM} \mathrm{MgCl}_{2}$ (Euroclone), $200 \mu \mathrm{M}$ each deoxynucleoside triphosphate (MBI Fermentas), $0.5 \mu \mathrm{M}$ of each of the 4 primers (MWG Biotech, HPSF grade), and $2.5 \mathrm{U}$ of taq polymerase (Euroclone). The sequence of the primers is described in elsewhere 
$[11,12]$. Then $5 \mu \mathrm{L}$ of DNA solution was used in the final reaction volume of 50 $\mu \mathrm{L}$. Amplification was done according to Harris [11], using the Mastercycler thermal cycler (Eppendorf). The amplification products were analysed using parametric analysis of gene set enrichment (PAGE), at 10\% concentration, stained with ethidium bromide, and visualized under UV light (312 nm). The modifications introduced were: the use of a DNA extraction kit, the inclusion of ammonium sulfate in the reaction mixture and the use of PAGE for band pattern analysis.

\section{Statistical analysis}

SPSS, version 12 was used for analysis. A confidence interval of 95\% (2-tailed) was applied.

\section{Results}

The 87 isolates were collected from 62 males, mean age 44 years (range 10-77 years), and 25 females, mean age 30 years (range 16-54 years). Most of the isolates came from patients resident in Mount Lebanon region (33\%) followed by Beirut (23\%), Tripoli (including
Doumia) (20\%), Akkar (10\%), the South (9\%) and Beqaa (5\% each). All isolates came from new cases of TB, except for 3 that were from previously treated cases.

The isolates included 21 resistant isolates $(24.1 \%)$ (18 primary-resistant): $4(4.6 \%)$ single-resistant (1R); 11 (12.6\%) double-resistant (2R),4(4.6\%) triple-resistant (3R); and 2 (2.3\%) quadruple-resistant (4R) (Table 1). A total of 8 (9.2\%) isolates were MDR; 3 of the MDR cases were the previously treated patients (3.5\%). The resistance pattern was: RIF 42,9\%, INH 81.0\%, EMB 19.1\%, STR 76.2\%.

The 87 isolates resulted in 17 different DRE-PCR patterns, while 12 isolates produced no detectable genotypes and were designated genotype $G x$ (Table 2 ). The diversity ratio (i.e. number of genotypes/number of isolates, excluding isolates with no genotype pattern detected) was therefore $0.23(17 / 75)$ (Table 1). A total of 9 clusters were observed, which included 67 isolates; 8 isolates had unique genotypes. The number of different bands was 14 and ranged from 1-3 for each genotype. The genotypes were assigned to 3 groups depending on the total number of bands (I-III). With 1 exception, all genotypes contained a $350 \mathrm{bp}$ band and, with 1 exception, all in genotype group III contained the same 2 bands (350 bp, 450 bp) (Table 2). The largest cluster was genotype 63 , containing the single band $350 \mathrm{bp}$, followed by cluster genotypes G9 and G12; together these represented $62 \%$ of the isolates (Table 2 ).

The profile of the 3 previously treated cases of TB were as follows: 1 male aged 41 years from Beqaa (genotype Gx, resistance pattern RRSS); 1 male aged 65 years from Beirut (genotype Gx, resistance pattern RRRR); 1 female aged 54 years from Beirut (genotype G1, resistance pattern RRSS).

\section{Correlations}

Various correlations between genotype, sex, age, resistance and region of collected isolates from the Lebanese population were investigated.

\section{Correlations related mainly to sex}

The general M:F ratio was $2.5(62 / 25)$ considering all isolates. For the Beirut region, it was $4.0(16 / 4)$, while the ratio for the regions outside Beirut taken together was $2.2(46 / 21)(\mathrm{OR}=1.83$,

\begin{tabular}{|c|c|c|c|c|c|c|c|}
\hline \multirow[t]{2}{*}{ Variable } & \multirow[t]{2}{*}{ Total } & \multicolumn{6}{|c|}{ Region } \\
\hline & & Beirut & Beqaa & South & Mount Lebanon & Tripoli & Akkar \\
\hline Total no. of isolates & 87 & 20 & 4 & 8 & 29 & 17 & 9 \\
\hline $\begin{array}{l}\text { No. of isolates in clu } \\
\text { genotypes }^{\text {a }}\end{array}$ & 67 & 12 & 3 & 8 & 23 & 13 & 8 \\
\hline $\begin{array}{l}\text { No. of genotypes } \\
\text { represented }\end{array}$ & 17 & 9 & 1 & 2 & 10 & 6 & 3 \\
\hline No. of resistant isola & & & & & & & \\
\hline All & $19+2 G x$ & $2+1 G x$ & $1 G x$ & - & 9 & 5 & 3 \\
\hline $1 \mathrm{R}$ & 4 & - & - & - & 1 & 3 & - \\
\hline $2 \mathrm{R}$ & 11 & 1 & 1 & - & 4 & 2 & 3 \\
\hline $3 R$ & 4 & 1 & - & - & 3 & - & - \\
\hline $4 R$ & 2 & 1 & - & - & 1 & - & - \\
\hline MDR & $6+2 G x$ & $2+1 G x$ & $1 G x$ & - & 4 & - & - \\
\hline Diversity ratio $^{\mathrm{b}}$ & 0.23 & 0.56 & 0.33 & 0.25 & 0.40 & 0.40 & 0.38 \\
\hline
\end{tabular}

$G x=$ no genotype pattern found; $-=$ no isolates.

$M D R=$ multidrug resistant.

$1 R=$ single-resistant $; 2 R=$ double-resistant, $3 R=$ triple-resistant $; 4 R=$ quadruple-resistant .

${ }^{a}$ Excluding unknown genotypes, $G x ;{ }^{b}$ Diversity ratio = no. of genotypes/no. of isolates (excluding $G x$ isolates). 


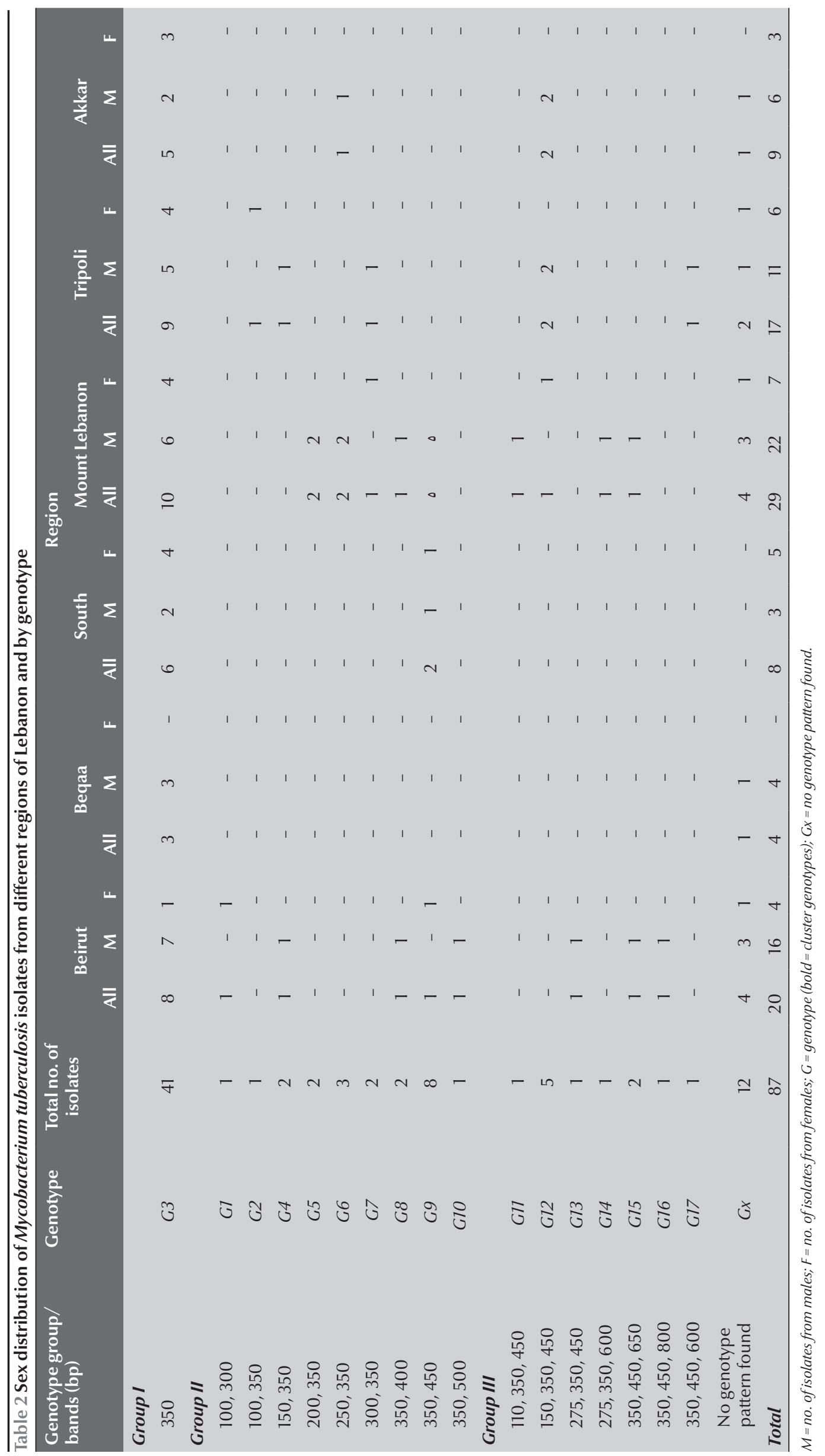


95\% CI: 0.54-6.14, $P=0.33$ ) (Table 2). The M:F ratio of cluster isolates was $2.4(47 / 20)$, while the ratio for unique genotypes was $3.0(6 / 2)$. The M:F ratio for resistant isolates was $2.5(15 / 6)$; however the specific genotype $G 3$ had 5 resistant isolates from females out of the total of 8 resistant isolates (Table 4). All MDR cases, except for 1 , were males, resulting in a M:F ratio of 7.0 $(7 / 1)(\mathrm{OR}=3.1,95 \% \mathrm{CI}: 1.27-7.39, \mathrm{P}$ $=0.3)$ (Table 3$)$. The isolates of patients aged 20-49 years produced a M:F ratio of $1.9(37 / 20)$, whereas the ratio for the rest of the age groups was $5.0(25 / 5)$. The M:F ratio was $11.6(25 / 16)$ for genotype group I (single band pattern $)(\mathrm{OR}=0.33,95 \% \mathrm{CI}: 0.11-0.97$, $P=0.029), 3.4(17 / 5)$ for genotype group II (2-band pattern) and 11.0 (11/1) for genotype group III (3-band pattern) $(\mathrm{OR}=5.5,95 \% \mathrm{CI}$ : 0.67-45.6, $P=0.11)$.

\section{Correlations related mainly to age}

A majority of isolates came from patients in the age range 20-49 years. This was mainly true in all regions, with female patients among the collected cases rarely affected after the age of 50 years. The diversity ratio of genotypes within the age range $20-49$ years was $0.22(12 / 54)$, excluding unknown genotypes, less than the diversity for the remaining age groups, $0.33(9 / 27)$. There was an apparent increase in the likelihood of resistance proportional to age, which was especially obvious for resistance to RIF (Table 4). We observed a greater tendency for the genotype group III to affect older people: 6/12 isolates were from patients in the age range $50-79$ years $(\mathrm{OR}=3.5,95 \% \mathrm{CI}$ : $0.98-12.45, P=0.055)$. All cases of MDR, defined as resistance to at least INH and RIF, were concentrated within patients aged 40-69 years, which was true for all genetic groups. Furthermore $75 \%$ (6/8) of MDR cases came from the age range 50-69 years (Table 4), even though only $19 \%$ of total isolates were from this age range.

\section{Correlations related mainly to geno- type and resistance}

The percentage of resistant isolates to total isolates was $24 \%(21 / 87): 25 \%$ (17/67) among clustered genotypes and $25 \%(2 / 8)$ for the unique genotypes (Table 4$)$. Nonresistant isolates had a diversity ratio of $0.20(13 / 66)$, while resistant ones had a value of 0.38 $(8 / 21)$. The diversity ratio for resistant isolates belonging to cluster genotypes (shown in bold font on Table 2) was $0.35(6 / 17)$ and for MDR isolates was $0.83(5 / 6)$. There were 4 genotypes (G1, G5, G15, G17) that appeared exclusively in resistant isolates (OR $=29,95 \%$ CI: 3.24-251.1, $P=0.003), 9$ genotypes (G2, G7, G8, G10, G11, G12, G13, G14, G16) appeared exclusively in nonresistant isolates and 4 genotypes (G3, G4, G6, G9) were shared.
Some genotypes exhibited no resistance to any drugs (e.g. none of the 5 isolates of G12), whereas for genotypes G15 and G5 drug resistance was demonstrated for all the collected isolates. The 2 isolates belonging to genotype G15 demonstrated MDR, while 12 other genotypes, representing 26 isolates, did not include any MDR cases $(\mathrm{OR}=34$, 95\% CI: 3.22-338.8, $P=0.004)$. These 2 isolates also showed identical resistance patterns (Table 4).

The percentage of resistant isolates was 20\% (8/41) for the genotype group I, and $36 \%(8 / 22)$ and $25 \%(3 / 12)$ respectively for the genotype groups II and III (Table 2). For STR resistance the proportion of resistant isolates decreased with increasing genotype group number $(\mathrm{I}>\mathrm{II}>\mathrm{III})$ whereas the opposite pattern was observed for RIF and INH resistance $(\mathrm{I}<\mathrm{II}<\mathrm{III})$ (Table 5). Most genotyped MDR cases (5 out of 6) belonged to genotype groups II and III, even though these represented only $45 \%(34 / 75)$ of all typed isolates (OR $=6.9,95 \%$ CI: $0.77-62.2, P=0.085)$ (Table 3).

\section{Correlations related mainly to region}

The ratio of resistant to total isolates in Beirut was 0.15 (3/20), while for outside Beirut it was $0.27(18 / 67)$ (OR $=0.48,95 \%$ CI: $0.126-1.82, P=0.085)$ (Table 1). Only a few cases of ENB resistance (16\% of resistant isolates) were observed (Table 5); and these

\begin{tabular}{|c|c|c|c|c|c|c|c|c|c|c|c|c|c|}
\hline \multirow[t]{3}{*}{ Region } & \multirow{3}{*}{$\begin{array}{l}\text { Total } \\
\text { no. of } \\
\text { isolates }\end{array}$} & \multicolumn{12}{|c|}{ Genotype group } \\
\hline & & \multicolumn{4}{|c|}{ I } & \multicolumn{4}{|c|}{ II } & \multicolumn{4}{|c|}{ III } \\
\hline & & All & $\mathbf{M}$ & $\mathbf{F}$ & MDR & All & $\mathbf{M}$ & $\mathbf{F}$ & MDR & All & $\mathbf{M}$ & $\mathbf{F}$ & MDR \\
\hline Beirut & 16 & 8 & 7 & 1 & 0 & 5 & 3 & 2 & 1 & 3 & 3 & 0 & 1 \\
\hline South & 8 & 6 & 2 & 4 & 0 & 2 & 1 & 1 & 0 & 0 & 0 & 0 & 0 \\
\hline Beqaа & 3 & 3 & 3 & & 0 & 0 & 0 & 0 & 0 & 0 & 0 & 0 & 0 \\
\hline M. Lebanon & 25 & 10 & 6 & 4 & 1 & 11 & 10 & 1 & 2 & 4 & 3 & 1 & 1 \\
\hline Tripoli & 15 & 9 & 5 & 4 & 0 & 3 & 2 & 1 & 0 & 3 & 3 & 0 & 0 \\
\hline Akkar & 8 & 5 & 2 & 3 & 0 & 1 & 1 & 0 & 0 & 2 & 2 & 0 & 0 \\
\hline Total & 75 & 41 & 25 & 16 & 1 & 22 & 17 & 5 & 3 & 12 & 11 & 1 & 2 \\
\hline
\end{tabular}

All = total no. of isolates in genotype group; $M D R=$ no. of multi-drug resistant isolates; $M=$ no. of isolates from males; $F=$ no. of isolates from females. 


\begin{tabular}{|c|c|c|c|c|c|c|c|c|c|c|}
\hline \multirow{3}{*}{$\begin{array}{l}\text { Age } \\
\text { group } \\
\text { (years) }\end{array}$} & \multicolumn{10}{|c|}{ Region } \\
\hline & \multicolumn{2}{|c|}{ Beirut } & \multicolumn{2}{|c|}{ Beqaa } & \multicolumn{2}{|c|}{ Mount Lebanon } & \multicolumn{2}{|c|}{ Tripoli } & \multicolumn{2}{|c|}{ Akkar } \\
\hline & Pattern & Gen.; Sex & Pattern & Gen.; Sex & Pattern & Gen.; Sex & Pattern & Gen.; Sex & Pattern & Gen.; Sex \\
\hline 10-19 & - & - & - & - & - & - & SSRS & $G 3 ; F$ & - & - \\
\hline \multirow[t]{3}{*}{$20-29$} & - & - & - & - & - & - & SSSR & $G 3 ; \mathrm{F}$ & SRSR & $G 3 ; \mathrm{F}$ \\
\hline & - & - & - & - & - & - & - & - & SRSR & $G 3 ; \mathrm{F}$ \\
\hline & - & - & - & - & - & - & - & - & SRSR & $G 6 ; M$ \\
\hline \multirow[t]{4}{*}{$30-39$} & - & - & - & - & SRSR & G3; M & - & - & - & - \\
\hline & - & - & - & - & RSSR & $G 3 ; M$ & - & - & - & - \\
\hline & - & - & - & - & SRSR & $G 5 ; M$ & - & - & - & - \\
\hline & - & - & - & - & SSSR & G9; M & - & - & - & - \\
\hline \multirow[t]{3}{*}{$40-49$} & - & - & RRSS & $G x ; M$ & RRRR & $G 5 ; M$ & SRSR & $G 3 ; \mathrm{F}$ & - & - \\
\hline & - & - & - & - & SRRR & G9; M & SRSR & $G 4 ; M$ & - & - \\
\hline & - & - & - & - & - & - & SRSS & G17; M & - & - \\
\hline \multirow[t]{2}{*}{ 50-59 } & RRSS & $G 1 ; \mathrm{F}$ & - & - & RRSR & $G 3 ; M$ & - & - & - & - \\
\hline & RRSR & G15; M & - & - & - & - & - & - & - & - \\
\hline \multirow[t]{2}{*}{$60-69$} & RRRR & $G x ; M$ & - & - & RRSS & $G 6 ; M$ & - & - & - & - \\
\hline & - & - & - & - & RRSR & G15; M & - & - & - & - \\
\hline 70-79 & - & - & - & - & - & - & - & - & - & - \\
\hline
\end{tabular}

Antibiotic resistance pattern sequence: rifampin (RIF)-isoniazid (INH)-ethambutol (EMB)-streptomycin (STR). $R=$ resistant; $S=$ sensitive.

Gen. = genotype; $G x=$ no genotype pattern found; $M=$ isolates from males; $F=$ isolatea from females; $-=$ no isolates

were restricted to cases from the Mount Lebanon and Tripoli area. Most forms of resistance to drugs involved INH and STR (each covered $79 \%$ of resistant genotyped isolates) (Table 4). Of the total of 21 resistant isolates, 17 resistance patterns were observed more than once and 9 of these 17 isolates also came from the same regions. However, only 2 of the 9 isolates resulted in the same genotypes (2 were genotype G3, both showing resistance pattern SRSR). We found 4 isolates of identical genotype and resistance pattern (genotype G3, resistance pattern SRSR) that were distributed in 3 regions (Mount Lebanon, Tripoli and Akkar) (Table 4). The various regions demonstrated similar levels of genotype diversity. The diversity ratio of genotypes for Beirut was $0.56(9 / 16)$ and for outside Beirut was 0.22 (13/59) (Table 1).

Mount Lebanon alone included $34 \%(23 / 67)$ of all cluster isolates (Table 2). All the 9 cluster genotypes, except $G 4$, included isolates of patients from Mount Lebanon, while the remaining 8 unique genotypes included only 2 from the Mount Lebanon area. The cluster genotypes could be divided into 2 categories: clusters in which several isolates belonged to the same region (G3, G6, G9, G12) and clusters that had single isolates belonging to different regions (G4, G7, G8, G15). In the second category all cases involved 1 isolate from Beirut or Mount Lebanon and Tripoli (Table 4); this category also demonstrated a deviation for the

\begin{tabular}{|c|c|c|c|c|c|c|c|c|c|c|c|c|c|c|}
\hline \multirow{3}{*}{$\begin{array}{l}\text { Genotype } \\
\text { group }\end{array}$} & \multirow{2}{*}{\multicolumn{2}{|c|}{$\begin{array}{l}\text { No. of resistant } \\
\text { isolates }\end{array}$}} & \multirow{2}{*}{\multicolumn{4}{|c|}{$\begin{array}{l}\text { No. of resistances } \\
\text { in each isolate }\end{array}$}} & \multicolumn{8}{|c|}{ Antibiotic resistances } \\
\hline & & & & & & & \multicolumn{2}{|c|}{ RIF } & \multicolumn{2}{|c|}{ INH } & \multicolumn{2}{|c|}{ EMB } & \multicolumn{2}{|c|}{ STR } \\
\hline & M & $\mathbf{F}$ & 1R & $2 \mathbf{R}$ & $3 \mathbf{R}$ & $4 R$ & No. & $\%$ & No. & $\%$ & No. & $\%$ & No. & $\%$ \\
\hline I & 3 & 5 & 2 & 5 & 1 & - & 2 & 25 & 5 & 63 & 1 & 13 & 7 & 88 \\
\hline II & 7 & 1 & 1 & 5 & 1 & 1 & 3 & 38 & 7 & 88 & 2 & 25 & 6 & 75 \\
\hline III & 3 & 0 & 1 & - & 2 & - & 2 & 67 & 3 & 100 & 0 & 0 & 2 & 67 \\
\hline Total & 13 & 6 & 4 & 10 & 4 & 1 & 7 & 37 & 15 & 79 & 3 & 16 & 15 & 79 \\
\hline
\end{tabular}

RIF = rifampin, INH = isoniazid, EMB = ethambutol, STR = streptomycin .

$M=$ isolates from males; $F=$ isolates from females.

$1 R=$ single-resistant $; 2 R=$ double-resistant, $3 R$ =triple-resistant $; 4 R=$ quadruple-resistant . 
M:F ratio (ratio 7, OR $=3.1,95 \% \mathrm{CI}$ : $0.36-26.3, P=0.31)$.

\section{Discussion}

The well-documented gender disparity for reported TB has been attributed to socioeconomic and cultural barriers in access to health care [13] as well as biological and epidemiological characteristics $[14,15]$. In this study the higher ratio of males to females that we detected shifted even more towards males when only MDR cases were considered (7:1). Male patients appeared more likely either to be infected by MDR strains or to develop MDR. The apparent targeting of males was also confirmed by the very high ratio of males to females $(10: 1)$ in isolates belonging to genotypes that were only observed within the age range 20-49 years. The sex ratio for cases from Beirut differed from those from outside Beirut. This, however, is consistent with the prevalence of genotype group I, which had a lower male to female ratio outside Beirut. In general the sex ratio-which may demonstrate a host-sex preference of M. tuberculosis TB — was an average of dissimilar ratios that depended heavily on the genotype of the M. tuberculosis TB strains. This was clearly shown when these ratios were calculated individually for each separate genotype group. The clear variation in host-sex preference according to genotype strain holds the potential for determining the genetic factors within $M$. tuberculosis TB that may underlie this phenomenon.

Between July 2002 and April 2004 Araj et al. studied the sensitivity of isolates from Lebanon and found 23.8\% resistant isolates, almost identical to the $24.1 \%$ obtained in the current study $[15,16]$. The regional distribution of drug resistance varies most clearly with regard to MDR strains, which were concentrated in the Mount Lebanon area and neighbouring Beirut. The latter was not the case considering resistant strains in general. Again, MDR strains did not behave like the bulk of resistant strains. Some forms of resistance were more common than others. The resistance pattern was dominated by INH and STR resistance, while few isolates showed resistance to ENB. The 4 isolates with identical resistance and genotype patterns are likely candidates for transmission of resistant strains of M. tuberculosis. It was noted that all these strains shared a common resistance to INH and STR. Considering that the underlying genetics of resistance are important for transmission dynamics [17]; this might affect the distribution of resistance patterns in cluster isolates.

In the present study in Lebanon, most $\mathrm{TB}$ patients were young adults; $72 \%$ were below the age of 50 years and $7 \%$ were aged 20 years or less. It appears that several genotypes may be restricted to, or favour, certain age ranges. Some genotypes appeared only in the age range where most cases of TB were obtained, while others were seen only at age ranges where there were fewer isolates. The genotypes belonging to genotype group III showed a tendency to affect older people, a finding which, if confirmed, could indicate the presence of genetic factors within the strains that are associated with age susceptibility of the host [18]. The increased occurrence of specific resistance depending on age was consistent with the elderly patients being most affected by MDR strains. An explanation for that shift might be that the elderly are generally too weak to fight successfully against the less fit resistant MTB strains [19].

Genotype groups appeared to be slightly selective regarding the pattern of drug resistance. The increase in the relative occurrence of RIF and INH resistance from genotype group I through to genotype group III is also consistent with the finding that most cases of MDR belonged to genotype groups II and III. The underlying genetic cause of these groupings and the shifts towards specific genotype groups (equivalent to a higher number of bands) could be a consequence of the possible increase in the numbers of the mobile genetic element IS6110; such an increase has been implicated or suspected in genotypic instability that could be associated with drug resistance and adaptation [20].

The higher diversity ratio of resistant isolates $0.42(8 / 19)$ compared with nonresistant ones $0.23(13 / 56)$ and the high ratio seen for MDR isolates $0.83(5 / 6)$ could be explained by the proposed lower level of relative fitness of many resistant isolates [17]. Cluster formation of genotypes was seemingly not related to the presence or absence of resistance. However, at the level of individual genotypes, some seemed to be more prone to resistance acquisition, persistence or spread than others.

Lebanon is considered an endemic country for $\mathrm{TB}$, with a general incidence estimate for 2005 of 11 per 100000 for all cases. However, the geographic distribution of the disease varies depending on the region. Studies presented by the Ministry of Health indicate the highest ratio of cases is in the regions of Beirut and the North (Tripoli and Akkar regions) [21].

The observed similarities in the patterns suggested a close relationship between most strains. However, the current study demonstrated that various genotypes tended to be more common in specific regions than others. This selective distribution of genotype groups indicates some level of geographic isolation and hence separate evolution. It justifies also the initial assignment of the genotypes to major groups depending on the general band pattern.

Earlier concerns about a large TB reservoir existing in the larger area of Beirut (which would include many former inhabitants of Mount Lebanon) [21] appear to be validated by 
the presence of isolates from this area in all genotypes forming clusters, suggesting an important role for the active spread of TB in Lebanon. The increased relative diversity of genotypes present in the region of Beirut compared with all regions outside Beirut as a whole seems to confirm the epidemic importance of this region, and its role as a major source for the spread of MTB strains.

\section{Conclusions}

The presented study demonstrates the importance of genotype characterization of MTB isolates incorporated within the essential framework of an adapted DOTS programme. Lowresource countries can still benefit from a plethora of available molecular techniques and adopt a programme that is relatively simple to implement and modest in its required resources. The widespread international incorporation of molecular strain differentiation into TB control programmes, associated with accurate epidemiological tracing, will provide a growing body of clinically applicable evidence. It may also shift the balance of control and cure in the battle against the growing threat of virulent MDR strains.
Acknowledgements

We were able to achieve this project owing to the financial support of the Lebanese University, the Syrian Ministry of Higher Education, represented by the Syrian High Council of Science, the Syrian National Commission of Biotechnology, and Al-Azm wa Al-Saadeh Society in Tripoli. We would like to express our appreciation to Professor Fawaz Al Azmeh, head of the NCBT, for his cooperation. We also thank Miss Sandy Nasr, Miss Zeina Merhi, Mrs Rana Nader, Mrs Mayssoun Elwi, Mrs. Nibal Qirahkahya, Miss Buthainah AlSalamah for their efforts.

\section{References}

1. Zigol $\mathrm{M}$ et al. Global incidence of multidrug-resistant tuberculosis. Journal of Infectious Diseases, 2006, 194:479-485.

2. Senol G, Komurcuoglu B, Komurcuoglu A. Drug resistance of Mycobacterium tuberculosis in Western Turkey: a retrospective study from 1100-bed teaching hospital. Journal of Infection, 2005, 50(4):306-311.

3. Verver $\mathrm{S}$ et al. Rate of reinfection tuberculosis after successful treatment is higher than rate of new tuberculosis. American Journal of Respiratory and Critical Care Medicine, 2005, 171:1430-1435.

4. Martínez M et al. Impact of laboratory cross-contamination on molecular epidemiology studies of tuberculosis. Journal of Clinical Microbiology, 2006, 44(8):2967-2969.

5. European Concerted Action on New Generation Genetic Markers and Techniques for the Epidemiology and Contro of Tuberculosis. Beijing/W genotype Mycobacterium tuberculosis and drug resistance. Emerging Infectious Diseases, 2006, 2(5):736-743.

6. Clark $\mathrm{CM}$ et al. Universal genotyping in tuberculosis control program, New York City, 2001-2003. Emerging Infectious Diseases, 2006, 12(5):719-724.

7. Montoro E, Valdivia J, Leão SC. Molecular fingerprinting of Mycobacterium tuberculosis isolates obtained in Havana, Cuba, by IS6710 restriction fragment length polymorphism analysis and by the double-repetitive-element PCR method. Journal of Clinical Microbiology, 1998, 36:3099-3102.

8. Sola C et al. Spoligotyping followed by double-repetitiveelement PCR as a rapid alternative to IS6710 fingerprinting for epidemiological studies in tuberculosis. Journal of Clinical Microbiology, 1998, 36:1122-1124.

9. Zapata P, Arbeloa M, Aznar J. Evaluation of mycobacteria growth indicator tube (MGIT) for drug susceptibility testing of Mycobacterium tuberculosis inoculated from clinical specimens. Clinical Microbiology and Infection, 1999, 5:227-230.

10. Shinnick TM, Good RC. Mycobacterial taxonomy. European Journal of Clinical Microbiology \& Infectious Diseases, 1994, 13(11):884-901.
11. Harris EA, Kadir N, eds. A low-cost approach to PCR. Appropriate transfer of biomolecular techniques. Oxford, Oxford University Press, 1998.

12. Friedman CD et al. Double-repetitive-element PCR method for subtyping Mycobacterium tuberculosis clinical isolates. Journal of Clinical Microbiology, 1995, 33:1383-1384.

13. Karim F et al. Gender differences in delays in diagnosis and treatment of tuberculosis. Health Policy and Planning, 2007, 22(5):329-334.

14. Hamid Salim MA et al. Gender differences in tuberculosis: a prevalence survey done in Bangladesh. International Journal of Tuberculosis and Lung Disease, 2004, 8(8):952-957.

15. Araj G, Saade H, Itani L. Nationwide study of drug resistance among acid-fast baulle positive pulmonary tuberculosis cases in Lebanon. International Journal of Tuberculosis and Lung Disease, 2006, 10(1):63-67.

16. Hamze M, Araj G. Drug resistance among mycobacterium tuberculosis isolates in Lebanon. International Journal of Tuberculosis and Lung Disease, 1997, 1(4):314-318.

17. Gagneux $S$ et al. Impact of bacterial genetics on the transmission of isoniazid-resistant Mycobacterium tuberculosis. PLOS Pathogens, 2006, 2(6):603-610.

18. McShane H. Susceptibility to tuberculosis-the importance of the pathogen as well as the host. Clinical and Experimental Immunology, 2003, 133:20-21.

19. Andersson DI. The biological cost of mutational antibiotic resistance: any practical conclusions? Current Opinion in Microbiology, 2006, 9:461-465.

20. 20. Tanaka MM, Rosenberg NA, Small PM, The control of copy number of IS6710 in Mycobacterium tuberculosis. Molecular Biology and Evolution, 2004, 21:2195-2201.

21. 21. Kalaajieh WK. Epidemiology of tuberculosis in Lebanon. International Journal of Tuberculosis and Lung Disease, 1999, 3(9):774-777. 